\title{
OPTIMALIZATION OF PHOTOREACTOR GEOMETRY FOR THE CULTIVATION OF CHLAMYDOMONAS REINHARDTII
}

\author{
Roman FeKete $^{a, b, *}$, Terézia ŽÁKová ${ }^{b}$, Luddmila Gabrišová $a^{a}$, \\ Peter Kotora $^{a}$, Peter Peciar ${ }^{a, b}$, Dominika Gahurová ${ }^{c}$, \\ Miroslava Slaninovác
}

${ }^{a}$ Slovak University of Technology in Bratislava, Faculty of Mechanical Engineering, Institute of Process Engineering, Námestie Slobody 17, 812 31, Bratislava, Slovakia

${ }^{b}$ Czech Technical University in Prague, Faculty of Mechanical Engineering, Department of Process Engineering, Technická 4, 16607 Praha 6, Czech Republic

${ }^{c}$ Comenius University in Bratislava, Faculty of Natural Sciences, Department of Genetics, Illkovičova 6, 842 15, Bratislava, Slovakia

* corresponding author: roman.fekete@stuba.sk

Abstract. At the present time, a great attention is being paid to the use of algae. Algae can adapt to different conditions and can produce substances corresponding to responsible environments. The main problem in their cultivation is the design of a suitable photoreactor. It should create the optimal conditions for their growth, which is mainly dependent on the contact of the algae with the light. The intensity of the light depends on the hydrodynamic conditions in the photoreactor and on its geometry. This paper deals with the study of kinetics of growth and gross biomass yield of biomass in laboratory photobioreactors, respecting their geometrical similarity as a basis for a possible scale-up. An optimal ratio between biomass growth rate and its gross biomass yield as a function of the photoreactor geometry is searched. Chlamydomonas reinhardtii were used as the model organism.

Keywords: algae; Chlamydomonas reinhardtii; scale up; photobioreactor; growth rate; gross biomass yield.

\section{INTRODUCTION}

Algae are very interesting microorganisms. Under the conditions of an environment, they can adjust their life cycle to produce various substances. Global warming, caused mainly by greenhouse gases, such as carbon dioxide, nitrious oxide and methane, is a current topic. One of the options for an effective reduction of carbon dioxide emissions is the usage of algae, which mediate the conversion of carbon dioxide to oxygen and other substances in dependence on the environment [1].

The commercial cultivation of algae for a production of organic bioproducts started in 1960s and early 1970s with the Chlorella (genus green alga) and Spirulina (cyanobacteria), followed by the production of betacarotene from alga Dunaliella salina since the 1980s [2. All these species are successfully grown in stirred or non-stirred open pools. The ability to grow in a highly selective media is the main reason for the successful growth of these species. Other species that are not able to adapt to specific conditions must be grown in closed photoreactors 3 .

Micro-algae have a lot of advantages, for example:

- their growth can be up to 10 times faster than a plant growth;

- soil and nutrients are not necessary for cultivation, it takes place in a fresh or saline water, and therefore algae do not compete with agricultural crops;
- the cultivation and processing of algae can be carried out all year long;

- the composition of substances produced by algae can be affected by changing conditions of the environment (e.g., in the absence of sulfur, the algae Chlamydomonas reinhardtii releases hydrogen instead of oxygen [4]);

- the possibility to obtain a relatively high concentration of selected valuable products (proteins, lipids, hydrocarbons, vitamins, food supplements, pigments, energy products, etc.) from various algae species;

- the utilization of carbon dioxide provides the possibility to use flue gases as a carbon source for increasing algal productivity and cleaning the atmosphere;

- algae can be processed into valuable raw materials for the production of, e.g., bio-diesel, bio-butanol, methane, ethanol and others [3].

Proteins are extremely important for human nutrition. Their lack is one of the most important malnutrition factors. Some algae contain up to $60 \%$ of the proteins. Algae, together with cyanobacteria, are also used for the production of a wide range of drugs, vitamins, vaccines, nutraceuticals and other 
nutrients. Algae and cyanobacteria contain various pigments such as chlorophyll and important phycobiliproteins and carotenoids with the potential to protect against sunlight. Furthermore, algae are a source of polyunsaturated fatty acids (also known as omega-3 or omega-6 fatty acids) [5]. Several species of Chlorella are important from the medical point of view as active immunostimulants. They reduce the effect of free radicals, reduce blood lipids, they are effective against gastric ulcers, wounds, odors and they prevent atherosclerosis and cancer [6]. Algae are a valuable source of almost all important vitamins including B1, B2, B6, B12, nicotinate, biotin, folic acid and pantothenic acid [6, 7].

Some micro-algae and cyanobacteria (Chlorella, Scenedesmus or Spirulina) are also suitable as feed additives for animals. These supplements increase immunity, fertility and bring many other health benefits [6, 8]. However, a long-term animal feeding by cyanobacteria can be harmful [6. They can be further used as a feed in aquatic cultures where micro-algae are the natural food 9 .

Micro-algae are flexible organisms with a wide application. However, for their optimal cultivation, it is necessary to select a suitable device. That is a photoreactor, which creates the optimal conditions for their growth rate and the production of the desired substances.

In practice, three basic technologies for the cultivation of algae are used: the phototrophic cultivation in open or closed tanks and heterotrophic cultivation in the closed vessels. In terms of construction, photobioreactors are basically either low-flowing fluid reservoirs or systems of thin-walled transparent tubes or sheets [10].

The choice of the appropriate type of photoreactor is not problematic. It is possible to perform experiments with a particular organism under laboratory conditions to optimize the composition of the culture medium, the light regime and the hydrodynamics of the culture medium to examine the kinetics of microorganism growth, the yield of the desired product or biomass itself, etc. The problem arises when these results need to be applied to operating devices that are of larger dimensions, which means the scale-up process itself is not examined in detail. It is the result of an enormous increase of the liquid culture medium volume. This causes, for example, increases of hydrostatic pressure and shear forces due to a different dynamics of liquid and turbulences caused e.g. by stirrers, pumps, etc. These parameters will be different than in laboratory devices. It creates other conditions for the transfer of a gas phase, cell diffusion, other light conditions, etc.

The scale-up methodology of photoreactors is a separate problem. As far as bio-chemical processes, which also depend on photosynthesis, are concerned, this is a very broad issue with a huge variability in the interaction among all the parameters.

\section{THEORY}

One of the main issues of the industrial device design is how to apply the knowledge and results obtained on small, laboratory-sized plants to production technologies of significantly larger dimensions. In process engineering, the scale-up method is used. For its reliable application, it is necessary to carry out a considerable number of experiments that provide sufficient data to select the important process parameters. On the basis of predominantly dimensionless criteria, these are more or less successfully applied to scale-up.

The simplest application of this method is on a device that works with non-living substances. If this method is applied to a technology with a livingorganism, the range of parameters that affect the operation of such device increases. The issues become even more complicated when it involves an algae cultivation. When a fermenter also works with living organisms, a closed device in which it is possible to observe the technological discipline to keep the process parameters under control, for example temperature, heat and gas supply, etc. is used. In industrial photoreactors, this is quite a big problem. Temperature, additives, and gas inlet and outlet are only some of the basic parameters. The first problem is, for example, temperature, which varies during a day and within a year according to daily and annual cycles. The algae have a tolerance to fluctuating temperatures, but their activity is affected.

Another problem is the inlet and outlet of the gas phase. As the algae use, among other things, $\mathrm{CO}_{2}$ for their growth and propagation and also produce $\mathrm{O}_{2}$, it is necessary to provide $\mathrm{CO}_{2}$. It is optimal if the $\mathrm{CO}_{2}$ is supplied in such volume that it can be dissolved in a liquid and then gradually consumed by the algae. This is a problem, because the growth kinetics and $\mathrm{CO}_{2}$ consumption depend on many factors. This means that the rate of $\mathrm{CO}_{2}$ supply should change according to the other process parameters. Therefore, if it is a closed photobioreactor, $\mathrm{CO}_{2}$ can be added at multiple places in order to compensate for its consumption. A greater problem is $\mathrm{O}_{2}$. In the literature, it is reported that algae are capable of producing up to $300 \%$ of the amount of oxygen that can be dissolved in the liquid [11. However, the problem is that oxygen in large amounts acts as an inhibitor. Therefore, the continuous deaeration of closed photoreactors is required [12]. Furthermore, according to the validity of the Henry Law, the solubility of gases varies with temperature and pressure, which are also parameters affecting photoreactors. In the case of open tanks, the pressure is mainly brought about by the effect of hydrostatic pressure. In closed vessels, it is caused by the pumps that are needed to ensure the circulation. When $\mathrm{CO}_{2}$ is supplied in the air, the dissolved amount of $\mathrm{CO}_{2}$ compared to $\mathrm{O}_{2}$ is much smaller because its content in the atmosphere is very small and thus its partial pressure is low. A comparison of the solubility of $\mathrm{CO}_{2}$ and $\mathrm{O}_{2}$ at atmospheric pressure when they 


\begin{tabular}{lcc}
\hline & $\begin{array}{c}\text { Oxygen } \\
\mathrm{O}_{2}\left[\mathrm{mg} \mathrm{l}^{-1}\right]\end{array}$ & $\begin{array}{c}\text { Carbon dioxide } \\
\mathrm{CO}_{2}\left[\mathrm{mg} \mathrm{l}^{-1}\right]\end{array}$ \\
\hline $\begin{array}{l}\text { Air in water } \\
\text { at atmospheric } \\
\text { pressure [13] }\end{array}$ & 7.54 & 0.42 \\
\hline $\begin{array}{l}\text { Separated } \\
\text { gases [14] }\end{array}$ & 37.0 & 1240.0 \\
\hline
\end{tabular}

TABle 1. $\mathrm{O}_{2}$ and $\mathrm{CO}_{2}$ solubility at $30^{\circ} \mathrm{C}$ and $101325 \mathrm{~Pa}$, salinity $0.0 \%$.

are supplied as a mixture in air or as pure gases is shown in Table 1

Therefore, it would be preferable to supply $\mathrm{CO}_{2}$ as a stand-alone gas, separated from other gases in the air. For smaller experiments, $\mathrm{CO}_{2}$ can be used from a cylinder and for larger scale, an exploitation of pretreated (purified) flue gases is assumed. Otherwise, it would be too expensive to run such plant.

Probably the most important parameter is lighting. It is the basis of photosynthesis. Growth rate, assuming steady fluid dynamics and temperature, is the function of the light profile of the reactor and the light mode to which the cells are exposed. In an environment with a high concentration of algae, they themselves prevent the light absorption due to shading. These effects create a light profile inside the photoreactor. As a result, there are several zones with different levels of illumination. As long as the cells remain in different zones with different illumination, it is a function of fluid dynamics. In an optimal system with no other limiting factors, the availability of light determines the rate of photosynthesis and thus algae growth. However, it should be noted that excessive light can also be harmful, causing biomass growth to slow down.

The dependence of the specific growth rate $\mu$ on the incident photosynthetic radiation on the horizontal area per hour, I, has long received considerable attention. An overview of the results of the work of various authors in this area is given, for example, in Molina 11. Tamiya published one of the first models in 1953, a relationship for calculating the specific growth rate of algae $\mu$ as a function of their maximum growth rate $\mu_{\max }$ and the light factors affecting this growth rate:

$$
\mu=\frac{\alpha \mu_{\max } I}{\mu_{\max }+\alpha I}
$$

where $I$ is the incident photosynthetic radiation on the horizontal area per hour and $\alpha$ is the coefficient of the equation.

Parameter $I$ is a key element, but its determination is rather complicated because the incident light on the surface of the photoreactor at a given moment is distributed to its space differently due to the optical properties of the environment, the refraction of light when crossing different phase interfaces, etc.
Generally, a specific growth rate $\mu$ increases with increasing irradiation, resulting in a maximum specific growth rate $\mu_{\max }$ value. However, further rises in irradiation may slow the growth. This phenomenon is known as photoinhibition.

At present, there are several approaches to assessing the impact of incident radiation and its impact on the algae growth. The general principle can be briefly summarized in a few short notes [11]:

(1.) Although the incident radiation from the outside is more or less constant, the irradiation within the culture changes as a function of the position. The cells near the surface of the photoreactor are exposed to higher irradiation than cells elsewhere in the vessel.

(2.) The mean irradiance value $I_{\mathrm{av}} 2$ is defined as the average amount of light received by an individual cell randomly moving inside the device.

For each photoreactor, the distance leading to a direct sunlight from the surface of the reactor to a certain point in the culture can be determined from the position of the sun, which determines the point of impact on the surface of the reactor and the polar coordinates $\left(r_{i}, \varphi\right)$. Where the distance is determined, the Beer-Lambert law for receiving local direct radiation $I_{\mathrm{Bt}}\left(r_{i}, \varphi\right)$ can be applied. Similarly, we obtain scattered local irradiation $I_{\mathrm{Dt}}\left(r_{i}, \varphi\right)$ :

$$
\begin{aligned}
I_{\mathrm{av}}= & \frac{1}{\pi R^{2}}\left(\int_{R} \int_{\varphi} I_{\mathrm{Bt}}\left(r_{i}, \varphi\right) r \mathrm{~d} r \mathrm{~d} \varphi\right. \\
& \left.+\frac{1}{2 \pi} \int_{R} \int_{\varphi} \int_{\varepsilon} I_{\mathrm{Dt}}\left(r_{i}, \varphi\right) r \mathrm{~d} r \mathrm{~d} \varphi \mathrm{d} \varepsilon\right),
\end{aligned}
$$

where the individual equation parameters determine the position of the reference point in the cylindrical coordinate system relative to the light source, for example, the sun [11].

(3.) When the cells are evenly distributed and evenly stirred, the average irradiation is again the same for all cells under these conditions. However, average irradiation is not a sufficient criterion for assessing the algae activity, as only the total length of the night and day periods, not the frequency with which the light-dark cycle changes, is considered.

(4.) Leaving aside the dynamics of cell movement, the degree of average irradiation inside the culture medium depends on the following factors: external irradiation at the surface of the reactor, reactor geometry, cell concentration and morphology, cell pigment grade, pigment absorption characteristics.

(5.) When the photoreactor is placed outside, in natural daylight, it is also necessary to take into account the cyclical changes in degrees of irradiance:

- a relatively long daily cycle,

- an even longer cycle based on changing seasons, 


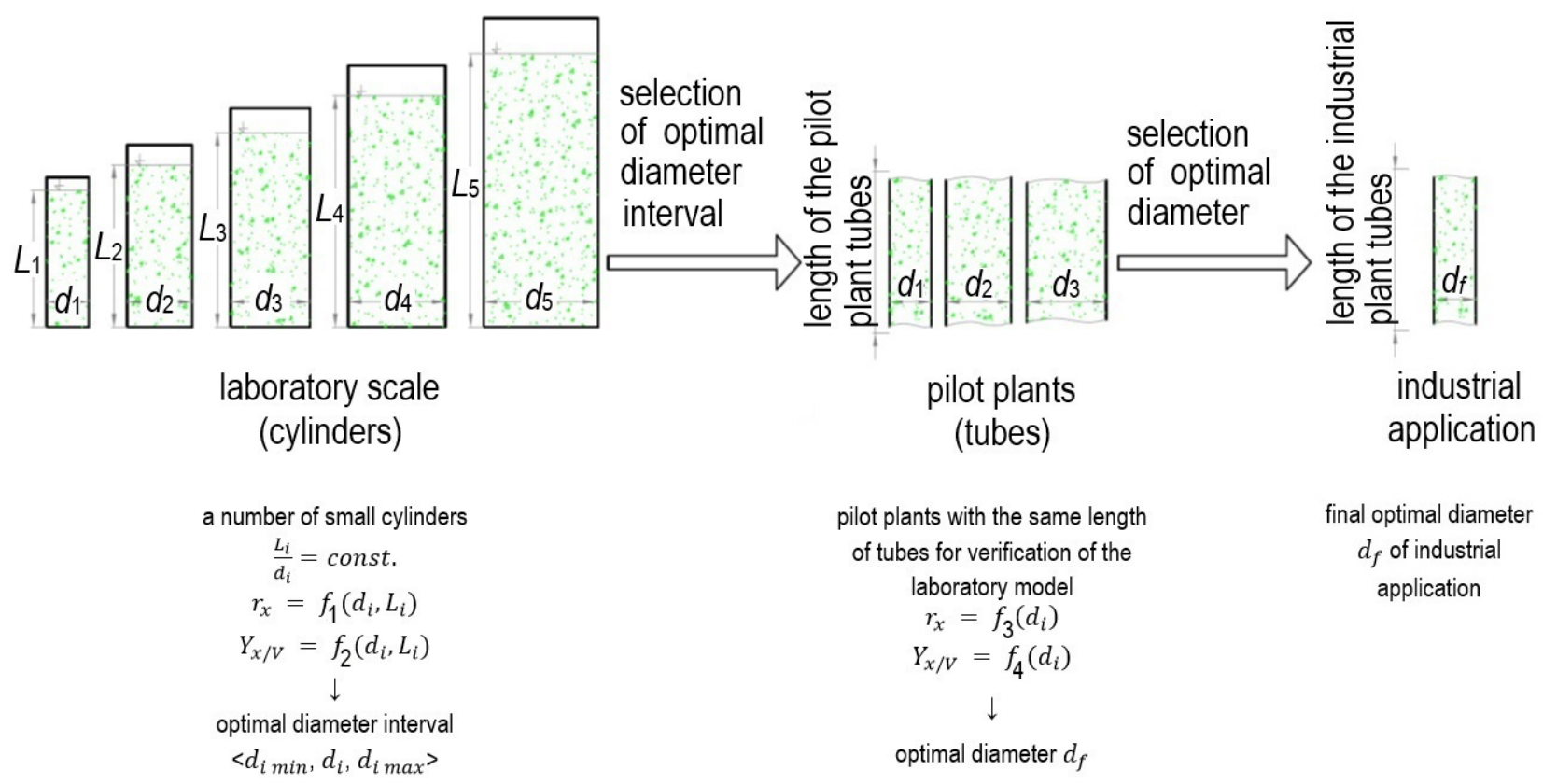

Figure 1. The key steps of scale-up for vessel design.

- that the cycle is a consequence of fluid movement between different light zones within the photoreactor.

The daily cycle means that the culture is limited by light at dawn and dusk. However, at noon, when the light is the strongest, the culture can be subjected to photoinhibition.

When the process is monitored in a specific device, these assumptions are sufficient. However, problems arise when scale-up engineering is applied.

The influence of the different photobioreactor geometry and the flow regime in its culture medium have an effect on the mean irradiance value $I_{\mathrm{av}} 2$ and thus also on the specific growth rate $\mu$ of the algae strain found in the plant and on the total productivity of the biomass in photobioreactor $P$.

\section{Methodology of EXPERIMEnts AND THEIR EVALUATION}

The experiments were focused on examining the mean irradiation value, $I_{\mathrm{av}}$, which is influenced by the photoreactor vessel geometry and the flow of the culture medium. One value is achieved for slim vessels with small diameters and heights, another for vessels where the ratio of diameter and height is almost equal. The experimental measurements were focused on these two geometric configurations.

It is not expected that the results obtained from the experiments will be used directly for the design of Chlamydomonas reinhardtii photoreactors. Their meaning is that they should provide an overview of how the specific growth rate and gross biomass yield vary as the dependence of the vessel geometry changes. The choice of a vessel geometry should indicate whether it is possible to optimize the ratio between the diameter of the tube and the volume per unit length of the photoreactor in relation to the specific growth rate and the gross biomass yield. The aim is to avoid the construction of pilot plant devices with a large amount of different tube diameters without a previous optimization in the laboratory scale. Based on this optimization, it would be possible to determine some tube diameters, e.g., one smaller and one larger than optimal. The next step would be to carry out experiments with pilot plant devices built from these tubes. The relationship between the tube diameter and gross biomass yield on a pilot plant scale would be obtained.

The natural light conditions should be preferred since artificial lighting is economically disadvantageous for large-volume applications.

(1.) The influence of photoreactor vessel geometry. The first series of experiments was focused on examining the geometric similarity of the photoreactors and their effect on the specific growth rate $\mu$ and the overall productivity of the biomass in the photobiorector $P$. The contents of the vessels were not mixed continuously but only twice in 24 hours to prevent an algae deposition on the vessel walls. The containers were chosen so that the ratio of vessel diameter $d$ and the height of the level $L$ was constant (Figure 2).

This vessel geometry replaces the one of the tubular photoreactors. The geometric similarity of the fillings in the vessels allows to assess the influence of the vessel diameter on biomass growth kinetics. The primary parameter is the vessel diameter, in the industrial application, it is the diameter of the photoreactor tubes as an element affecting the intensity of the illumination at the individual position of the 


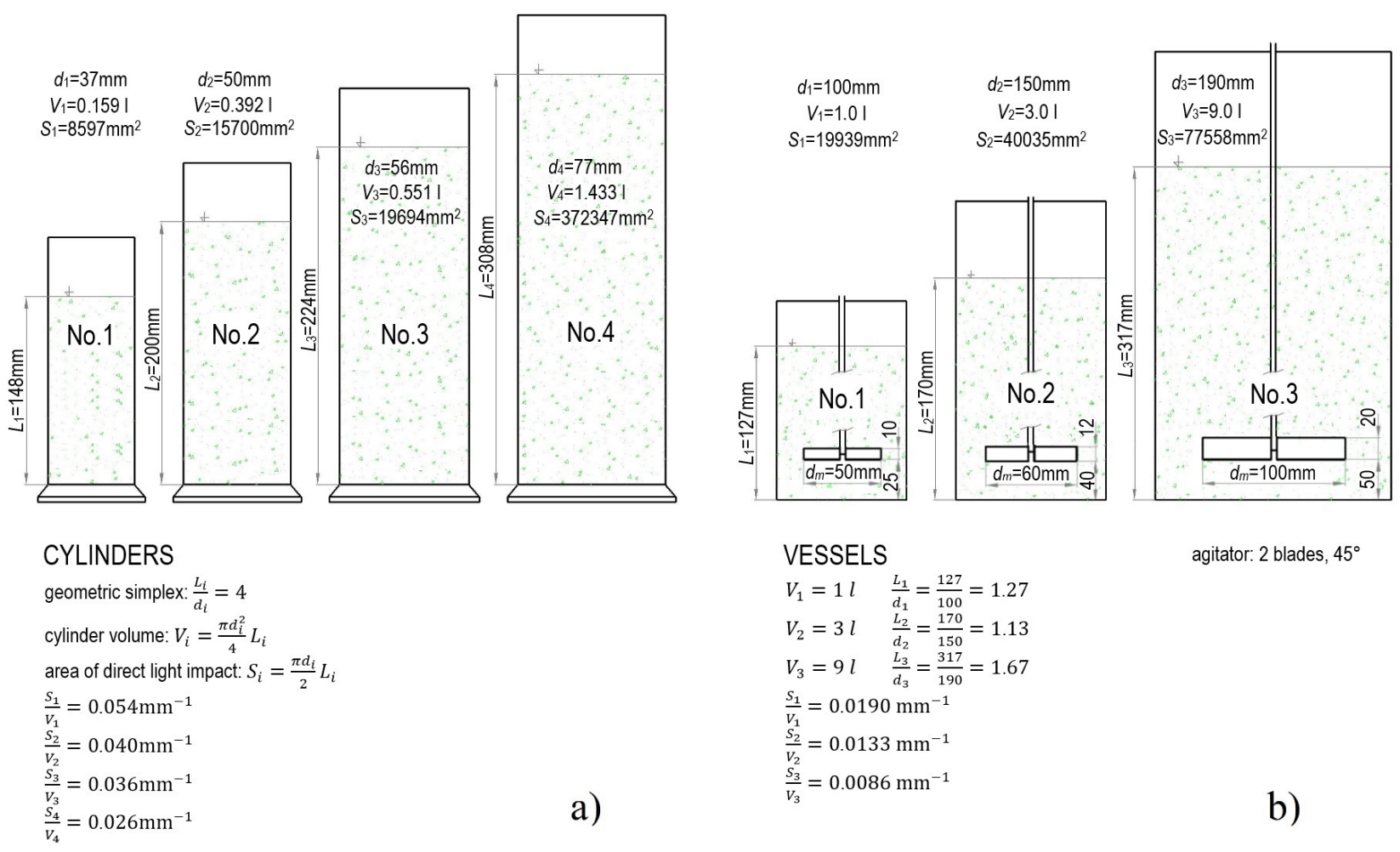

FiguRE 2. Geometry of cylinders and vessels and the comparative criteria for studying the shape and volume of the algae cultivation: a) the influence of the photoreactor vessel geometry; b) the influence of the photoreactor vessel volume.

photoreactor cross-section. The constant ratio between the vessel diameter and height of liquid level was selected. It allows to increase the length of the industrial photoreactor by multiplying the height of the liquid column in the laboratory model, while maintaining the diameter constant. This allows increasing of the volume of the industrial photoreactor while maintaining the same light intensity in the tube cross-section.

The vessels were not stirred because the flow in tubular photobiocrectors is influenced by hydrodynamic conditions. These are influenced by the effect of the pump, the geometry of the photoreactor, the number of elbows, the roughness and the length of the tubes, the various fittings, etc. Therefore, the mixing is different in each part of the photoreactor, due to the fact that the amount of light incident to the individual algae is irregular, depending on the location where the algae are present. This is shown, for example, by a relationship 2 2 The volume of the vessel was mixed 2 times in 24 hours to prevent algae settling on the bottom of the vessels and on the side facing away from the incident light.

(2.) The influence of photoreactor vessel volume. A second series of experiments was focused on examining the effect of the liquid volume, with the volume of the liquid medium of the following vessel being 3 times the previous vessel, i.e., 1 liter $/ 3$ liters $/ 9$ liters. The vessels were not con- tinuously mixed. The main task was to prevent an algae settlement at the bottom and on the walls. The stirring was switched on for 8 hours and for 16 hours, the vessels were not mixed.

This solution is similar to the one for various containers, bags, etc., but not to the tubular ones. In such devices, the conditions for spreading the light are much worse than in tubular devices. The mixing has the function of preventing sedimentation. At the same time, it conveys the individual algea between the side inclined to the light and the shadow. Mixing need not to be continuous. If algae use light, stirring is appropriate only during the hours when there is a sufficiency of light. Alternatively, the mixing can be switched off to reduce the operation cost of the photoreactors.

The Reynolds number at an average culture medium temperature of $25^{\circ} \mathrm{C}$ was:

$$
\begin{array}{r}
R e_{M}=\frac{n d_{m}^{2} \rho}{\eta}=\frac{2.5 \mathrm{~s}^{-1} \times(0.1 \mathrm{~m})^{2} \times 997 \mathrm{~kg} \mathrm{~m}^{-3}}{0.0009 \mathrm{Pas}} \\
=27694
\end{array}
$$

where $n$ is a stirrer speed, $d_{m}$ is a diameter of the agitator, $\rho$ is the water density, $\eta$ is the water dynamic viscosity, which is related to a starting condition in the vessels that is similar to the clean water properties. All the other agitators had the operating speed in accordance with their diameters in order to keep $R e_{M}$ constant. 


\begin{tabular}{|c|c|c|c|c|c|c|}
\hline \multirow[t]{2}{*}{ Time } & \multirow{2}{*}{\multicolumn{2}{|c|}{$\begin{array}{l}\text { Cloudiness } \\
\text { (number of } \\
\text { days during } \\
\text { experiment) }\end{array}$}} & \multicolumn{2}{|c|}{$\begin{array}{l}\text { Cylinder (Fig. } 6 \text { - distance } \\
\text { from the window } 0.3 \mathrm{~m}\end{array}$} & \multicolumn{2}{|c|}{$\begin{array}{l}\text { Vessel (Fig. } 7 \text { ) - distance } \\
\text { from the window } 0.8 \mathrm{~m}\end{array}$} \\
\hline & & & $\begin{array}{l}\text { Growth media } \\
\text { temperature }\left[{ }^{\circ} \mathrm{C}\right]\end{array}$ & $\begin{array}{l}\text { Exposure } \\
\text { value }[\mathrm{l}]\end{array}$ & $\begin{array}{l}\text { Growth media } \\
\text { temperature }\left[{ }^{\circ} \mathrm{C}\right]\end{array}$ & $\begin{array}{l}\text { Exposure } \\
\text { value }[\mathrm{lx}]\end{array}$ \\
\hline $8: 30$ & 2 days & 潆 & 27 & 1280 & 25 & 640 \\
\hline 16:00 & & & 36 & 112 & 31 & 56 \\
\hline $8: 30$ & 7 days & = & 21 & 450 & 21 & 320 \\
\hline 16:00 & & & 26 & 40 & 24 & 56 \\
\hline $8: 30$ & 5 days & 洸与 & 22 & 28 & 22 & 10 \\
\hline 16:00 & & & 25 & 40 & 24 & 40 \\
\hline $8: 30$ & 2 days & $3_{3}$ & 22 & 56 & 22 & 56 \\
\hline 16:00 & & & 26 & 28 & 24 & 28 \\
\hline
\end{tabular}

TABLE 2. Light conditions during experiments [21].
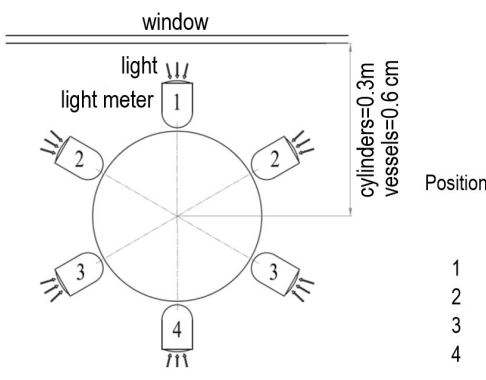

Vessel
illuminance
$[$ [Ix]
56
40
7
3.5

FiguRE 3. Light profile of cylinders and vessels measured by the light meter.

The results of the experiments were evaluated in relation to the geometry of the vessels and the volume of the culture medium $V_{i}$ to the biomass growth rate $r_{x}$ and the productivity of the biomass $P$ corresponding to a one litre of the volume of culture medium $Y_{x / V}$.

The calculation of the irradiation according to the equation 2 is almost impossible. The simpler method was chosen for these experiments. In the Figure 2 the size of the surface on which the light directly falls is stated and it is calculated as $50 \%$ of the surface area of the cylinder or vessel. This value was chosen based on the measurement of the incident light on the cylinder surface, as shown in Figure 3 Measurement was carried out with a light meter measuring the light impacting on the surface of the cylinder, every $45^{\circ}$. The measurement was done at $12.00 \mathrm{a} . \mathrm{m}$. with an overcast sky. The distance of the cylinders from the south-facing window was $0.3 \mathrm{~m}$. The distance from the window was $0.6 \mathrm{~m}$.

The vessels did not have a thermal control. The temperature was measured daily. Typical values for various degrees of cloudiness are shown in Table 2

\subsection{Strain OF Algae}

For the experiments, the green algae Chlamydomonas reinhardtii (strain 21gr) was used. It is unicellular eukaryotic microorganism with the cells about $10 \mu \mathrm{m}$ in diameter. The cells are the most commonly spherical or elliptical with two flagella and one chloroplast partially surrounding the cell nucleus. The haploid vegetative cells multiply by a mitotic division. They are found in wide range of environments all around the world, appearing mainly in soils and fresh water.

Chlamydomonas reinhardtii is a well-known model organism, also because of its simple cultivation and the ability to manipulate its all three genomes. The algae are photosynthetic organisms and for standard growing they need visible light, but, in the presence of acetate, most strains of Chlamydomonas can also grow in the dark [15].

These algae are commercially interesting primarily because of their application in pharmacy and also for the production of biofuels [16]. Under specific conditions, they can produce hydrogen instead of oxygen [17.

\subsection{Culture medium}

For cultivation of Chlamydomonas was used TAP medium with acetate which allows growth also in the dark [15, 17].

The culture medium TAP was selected for the cultivation in the natural daylight mode, 24 hours cycle. It consists of $50 \mathrm{ml}$ Beijerinck solution for TAP $\left(8 \mathrm{~g} \mathrm{NH}_{4} \mathrm{Cl}+1 \mathrm{~g} \mathrm{CaCl}_{2} .2 \mathrm{H}_{2} \mathrm{O}+12 \mathrm{~g} \mathrm{MgSO}_{4} .7 \mathrm{H}_{2} \mathrm{O}\right.$, fill up to $1000 \mathrm{ml}$ ), $3.75 \mathrm{ml}$ Phophate solution, $25 \mathrm{ml}$ Trisacetate solution ( $48.4 \mathrm{~g} \mathrm{C}_{4} \mathrm{H}_{11} \mathrm{NO}_{3}+20 \mathrm{ml} \mathrm{CH}_{3} \mathrm{CO}_{2} \mathrm{H}$ fill up to $1000 \mathrm{ml}$ ), $1 \mathrm{ml}$ Hutner's trace elements.

The aim of the measurements was to avoid the need of artificial lighting, since its application would mean additional costs for the operation of large photoreactors.

The experiments were carried out during April and May when the ratio of time between sunrise and sunset ranges from 12 to 16 hours. This indicates that, at night, the conditions for growth were heterotrophical and during the day autotrophical. This culture medium allows for such growth. If the growth is to be autotrophical, $\mathrm{CO}_{2}$ is needed. For these measure- 


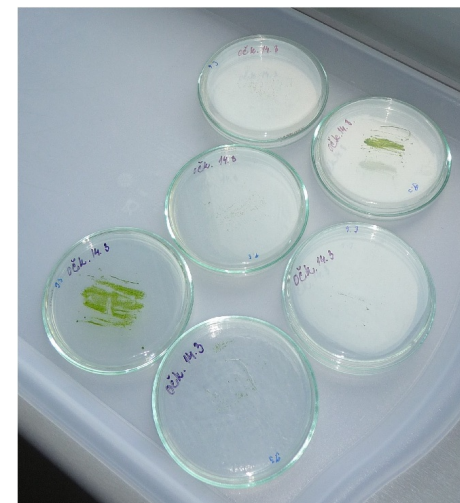

a)

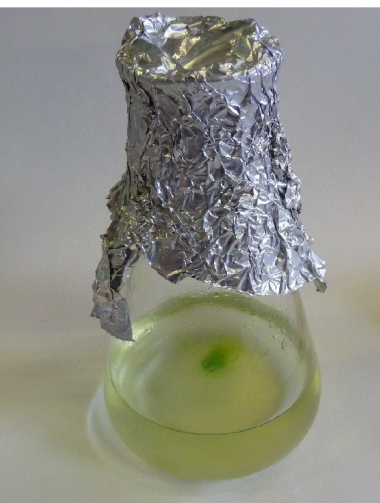

b)

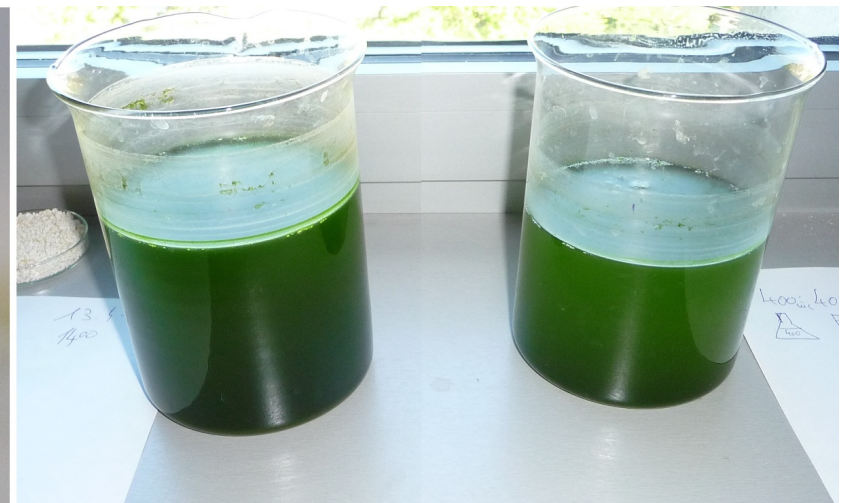

c)

Figure 4. Cultivation of algae: a) on nutrient agar after inoculation; b) release of algae from agar in an Erlenmeyer flask prior to application to culture medium in photoreactors; c) inoculum in seed photobioreactor vessels.

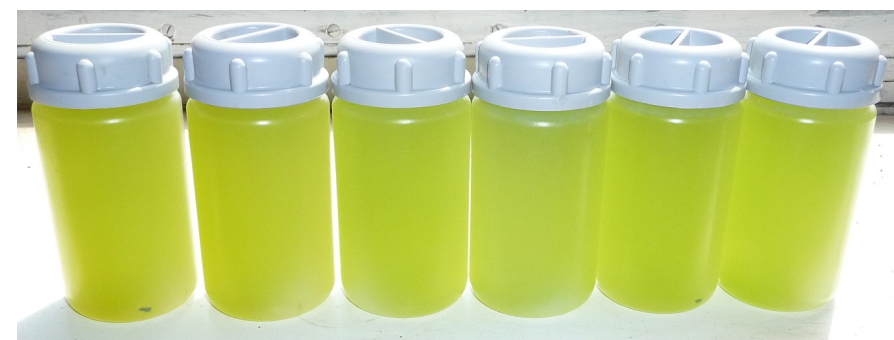

a)

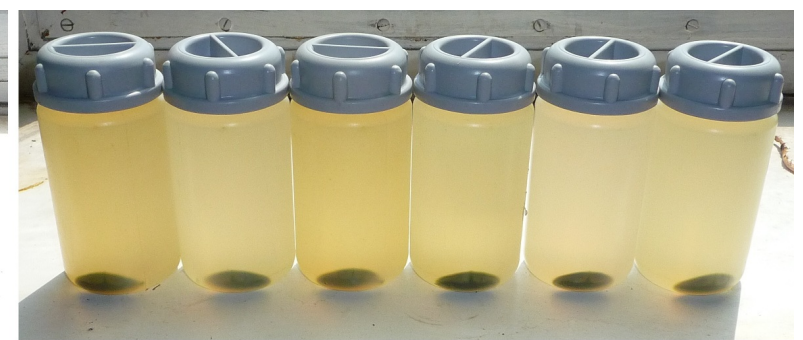

b)

FiguRE 5. Culture medium after cultivation: a) before centrifugation, b) after centrifugation.

ments, this parameter was left to the natural absorption properties of the culture medium. The $\mathrm{CO}_{2}$ was absorbed through the surface of the cultural medium in the cylinders and vessels. This is for several reasons. Vessels have different geometries so it is questionable how to set the aeration to preserve the similarities in terms of diffusion of air or $\mathrm{CO}_{2}$ in the culture medium. As the impact of photobioreactor geometry on light conditions and thus on the biomass growth kinetics was investigated, the impact of $\mathrm{CO}_{2}$ was not a subject of interest.

The experiments were realized in a south-facing area. Thus, the algae used the direct sunlight on the surface of the photoreactors for approximately 5 hours, then only the diffused light falling into the locality was used. The rest of the cultivation was in the dark. In Table 2 the light conditions during the experiments are shown.

Taking into account these facts, the TAP medium, the natural daylight cycle and the natural diffusion through the culture media layer would seem to be used optimally.

\subsection{Preparation of SAMPles}

The Chlamydomonas strain was cultivated after inoculation on a Petri dish with nutrient agar (Figure 4 a).

Before the experiments, the algae strain and the agar were removed from the Petri dish and put into a $200 \mathrm{ml}$ culture medium in an Erlenmeyer flask for several days (Figure $4 \mathrm{~b}$ ). Then, the content of the flask was poured into two vessels with a $750 \mathrm{ml}$ culture volume placed at a window on the northwest side of the building (Figure 4k). They were the seed photobiorectors, where the initial algae concentration was obtained after several days. Their content was mixed twice per day with a laboratory glass rod to prevent a settlement. Experiments required 15.5 liters of culture medium. It was created from two seed photobioreactors, with a total volume of 1.5 litres, and 14 liters of TAP medium that were poured into one container. Using a hemocytometer and a microscope, the initial algae concentration was determined. Its value was 290 cells $\mathrm{mm}^{-3}$ (Figure 8). This medium, with the initial concentration of algae, was gradually poured into individual photobiorectors (Figures 6 and 7 ).

\subsection{Growth Kinetics}

It was evaluated using the standard procedure used in cell experiments [18]. A hemocytometer and a microscope were used to determine the cell number per unit volume. A change in this parameter was then evaluated against time.

\subsection{Yield OF CULTIVATION}

The resulting yield was made by centrifuging the 200 $\mathrm{ml}$ of culture medium and separating the liquid phase and the sediment. The wet biomass was weighed, so the results refer to the wet biomass weight. This 


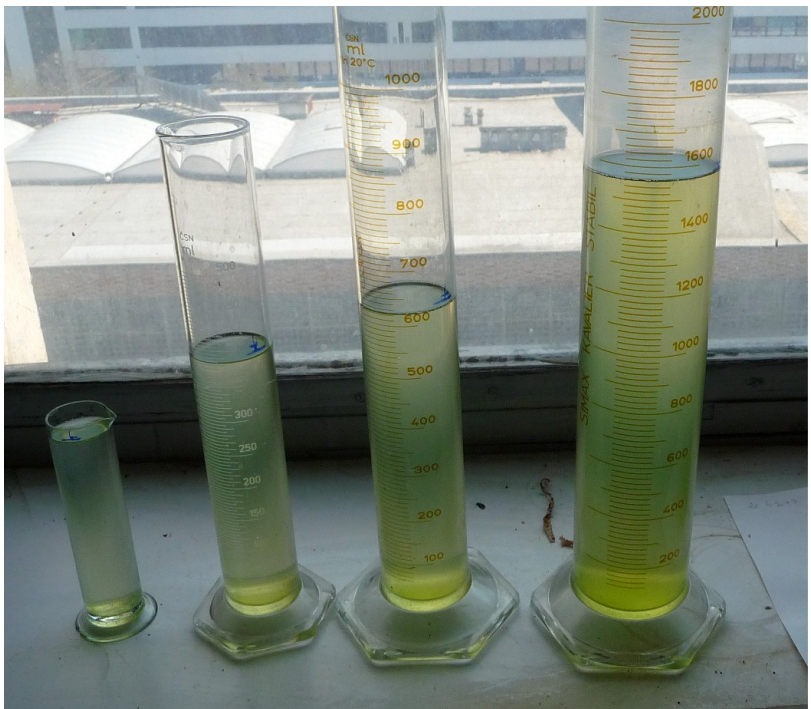

a)

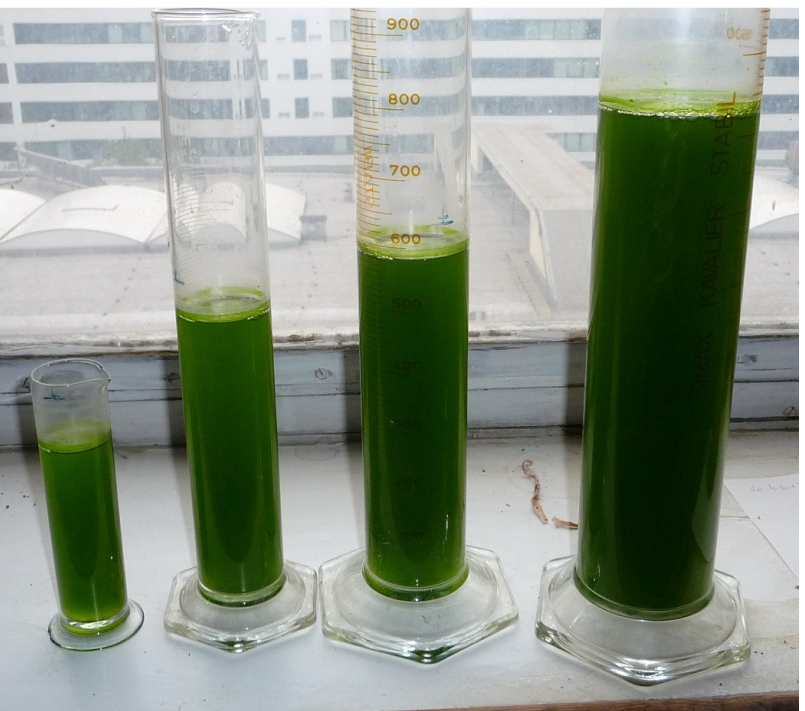

b)

FIGURE 6. Investigation of the influence of the geometrical similarity on algae growth rate: a) after filling the cylinders with the culture media with the initial concentration of algae 24. 04. 2017, the differences of green color saturation are due to the differing diameters of the cylinders; b) the color saturation on the fourth day of cultivation 28. 04. 2017.

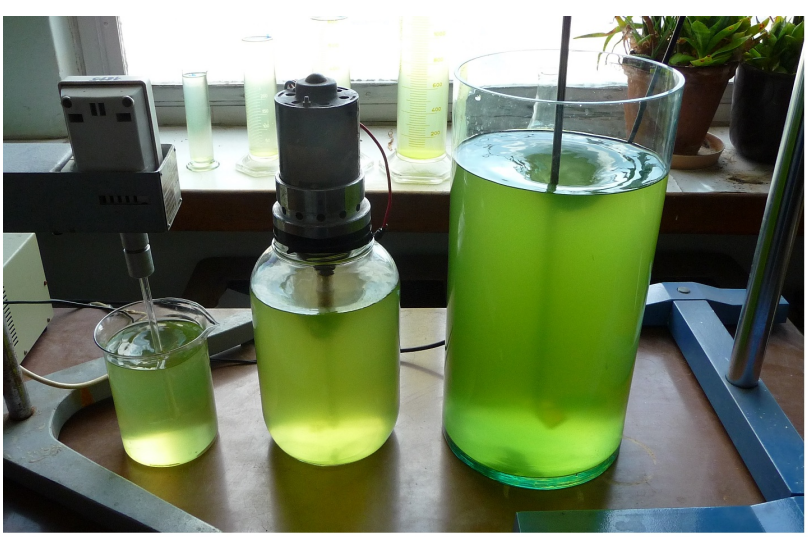

a)

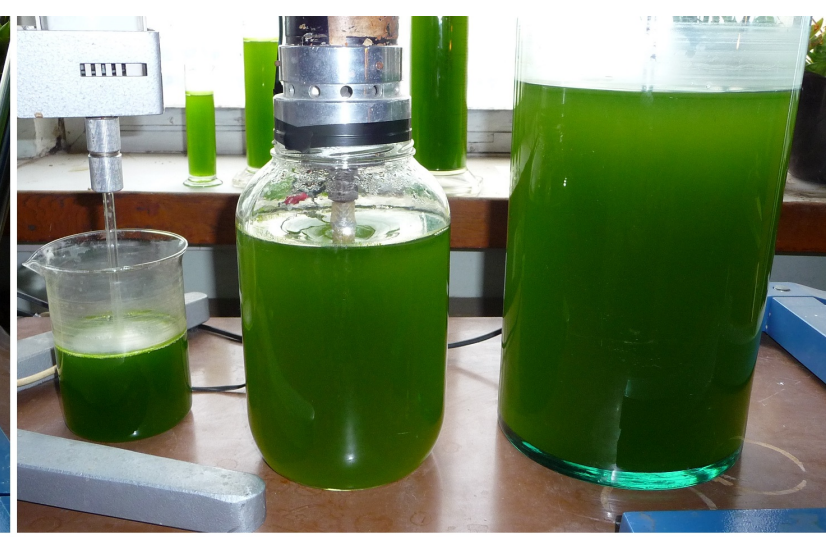

b)

Figure 7. Examining the influence of the volume of the culture medium on the growth rate of algae: a) after filling the vessels with a culture media with an initial concentration of algae 24. 04. 2017, the differences of green color saturation are due to the differing diameters of the vessels, b) the color saturation on the fourth day of cultivation 28. 04. 2017. Total volume of medium 1 liter, 3 liters, 9 liters.

parameter is often the input for the design of other industrial apparatus for the biomass processing, for example, the cells disruption. The various methods are used [19]. The disruption in the bead mill [20] requires, among other things, to known the feed rate and the cell concentration in the suspension. Therefore, it is advantageous to know the weight of the wet biomass. The influence of different cultivation parameters on the biomass composition, e.g. lipids, water etc. is, therefore, not the subject of these experiments.

\section{EXPERIMENT PROCEDURE}

The experiments were focused on the testing of a cultivation in a natural light mode similar to those the industrial photoreactor would also be exposed to.
The experiments were focused on two issues:

(1.) Examining the influence of the vessel geometry on the growth rate $r_{x}$ and yield $Y_{x / V}$ of the cultivation at a constant ratio of cylinder diameter and layer height, if the initial concentration of algea $x_{i}$ was the same. The top surface area of liquid, which depends on the $d_{i}$, will have the predicted effect. Through this area, $\mathrm{CO}_{2}$ is absorbed from the air. The diameter of the cylinders also affects the light intensity $I_{\text {av }}$ from the window towards the opposite side of the cylinder. This means that with the diameter $d_{i}$ increasing, the intensity of the light $I_{\mathrm{av}}$ decreases in a direction towards the rear of the cylinders. The height of the layer $L_{i}$ and the diameter $d_{i}$ influence the volume 
$V_{i}$ and thus the amount of the light $I_{\text {av }}$ that falls on a volume unit. Thus, the intensity of the light varies throughout the volume of the photoreactor, as described by the mean irradiance value $I_{\mathrm{av}}(2)$.

However, the application of this relationship is complicated. Therefore, a different benchmark was used. It is assumed that the highest intensity of the incident light, in the case of photobioreactors located at the window, is on the half of the surface area of the cylinders that is oriented towards the light source. The assumption is that the other half of the surface area is in a shadow, and thus the intensity of the incident light is insignificant. Therefore, a new comparison parameter is defined. It is the ratio of a half of the surface area of the culture medium $\mathrm{Si}$ to the volume of the culture medium $V_{i}$ in the cylinder. Figure 2 shows the dimensions and values of the comparing parameters. It can be seen that the ratio between the increasing volume $V_{i}$ and the area of direct light impact $S_{i}$ of the culture medium decreases as the volume of the cylinder increases. This means that the overall light intensity decreases with increasing volume. It is, therefore, possible to assume that the growth rate should be highest in the smallest cylinder No. 1 and lowest in the largest cylinder No. 4 (Figures 2 and 6 ).

The volume of the medium in the cylinders was stirred twice a day, at 8:00 a.m. and at 4:00 p.m., to prevent a settlement of the algae on the wall and bottom and to force them to hover.

The intensity of the incident light was measured continuously. The light intensity was measured by the exposure meter placed in position 1 (Figure 3). The light conditions are shown in Table 2 Samples of the culture media were taken regularly and the algae concentration was calculated in a hemocytometer.

(2.) Examining the effect of the vessel volume $V_{i}$ on the growth rate $r_{x}$ and yield $Y_{x / V}$ of the cultivation with the initial algae concentration $x_{i}$ being identical. Three vessels with three different $V_{i}$ volumes were used: 1.0 liter, 3.0 liters and 9.0 liters (Figures 2 and 7). The vessels were continuously stirred from $8.00 \mathrm{a} . \mathrm{m}$. to 4.00 p.m. The intensity of the incident light was measured continuously. Samples of culture media were taken regularly and the algae concentration was calculated in a hemocytometer.

\section{Results AND Discussions}

The results of experiments were processed in two ways.

(1.) Kinetics of biomass growth in cylinders and containers. The kinetics of biomass growth are defined by the growth rate [18]:

$$
r_{x}=\frac{\Delta x}{\Delta t}
$$

therefore, as a change in the cell concentration over time. Since the cultivation passes through different phases, that part of the measured data where the cell concentration changes was used to evaluate this parameter. It is the phase of the maximum growth rate of biomass $r_{x, \max }$ under the given conditions of the cultivation (Figure 8).

From the experimental data for individual cylinders (Figure $8 \mathrm{z}$ ) and for individual vessels (Figure 8p), the individual values of maximum growth rate $r_{x}$ of biomass were calculated according to the formula (4). The influence of the volume $V_{i}$ of the culture medium on the maximum growth rate $r_{x, \max }$ of the biomass is in (Figure 8p). Figure 8d shows the maximum growth rate $r_{x, \max }$ of the biomass in relation to the ratio of the illuminated surface $S_{i}$ to the volume of the vessel $V_{i}$ (Figure 22. The growth rate was determined from sampling and consequent loading into the hemocytometer. The point value was determined as the mean of four values of the $1 \times 1 \mathrm{~mm}$ hemocytometer field under a microscope. The gross biomass yield was determined from each vessel at the end of the experiment. The experiments were not repeated, as cultivation took place in the light at the end of April and the beginning of May, so it was not possible to ensure similar light conditions.

The influence of the volume $V_{i}$ on the growth rate $r_{x}$ of biomass has the same tendency for cylinders and for vessels. The growth rate $r_{x}$ of biomass decreases with the increasing volume $V_{i}$. The decrease is significantly higher in the cylinders, which may be due to the fact that the cylinders are not mixed. Because the amount of light intensity in the volume decreases with the increasing diameter $d_{i}$, the microorganisms that are on the opposite side of the cylinder do not have enough light and their growth is slower.

The decrease of the growth rate $r_{x}$ is not so distinctive in the vessels because they are stirred. This means that the biomass passes through zones with a varying intensity of light $I_{\mathrm{av}}$, which creates better conditions for its growth. However, the growth rate $r_{x}$ is much smaller, despite the stirring, because the intensity of the illumination $I_{\mathrm{av}}$ in the whole volume is not comparable to the light intensity in the cylinders. They have a smaller diameter $d_{i}$, and therefore the intensity of the illumination $I_{\mathrm{av}}$ in a volume $V_{i}$ is higher. However, it is still worth pointing out that this comparison is not entirely objective, since the relative position of the cylinders and vessels was such that the intensity of the vessel lighting was lower than that of the cylinders (Table 22. But the difference in the growth rates $r_{x, \max }$ has significantly greater variations. The main goal was not to compare these measurements to each other, but to evaluate them separately.

An interesting comparison is the relationship between the ratio of the area of the direct light incident 


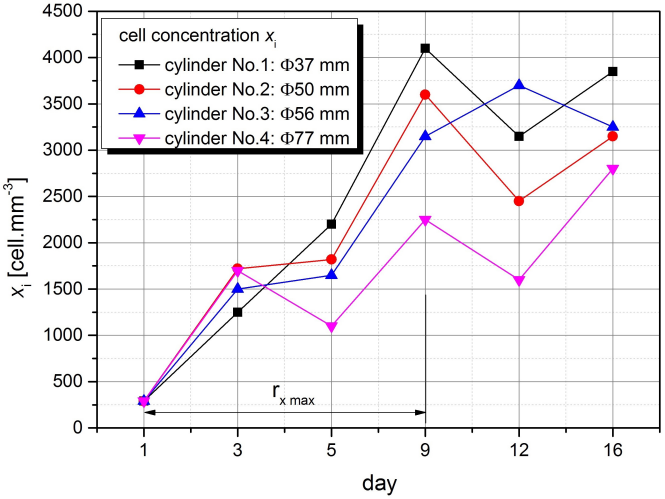

a)

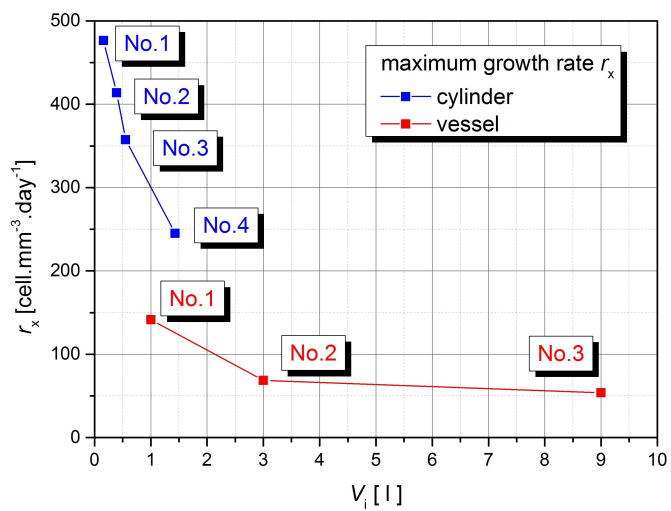

c)

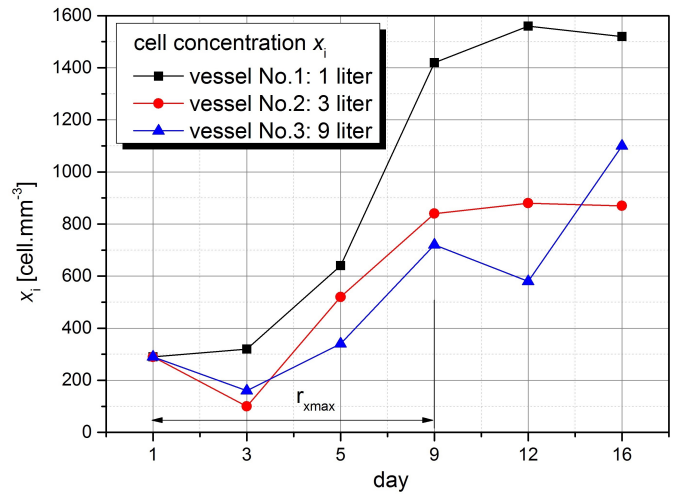

b)

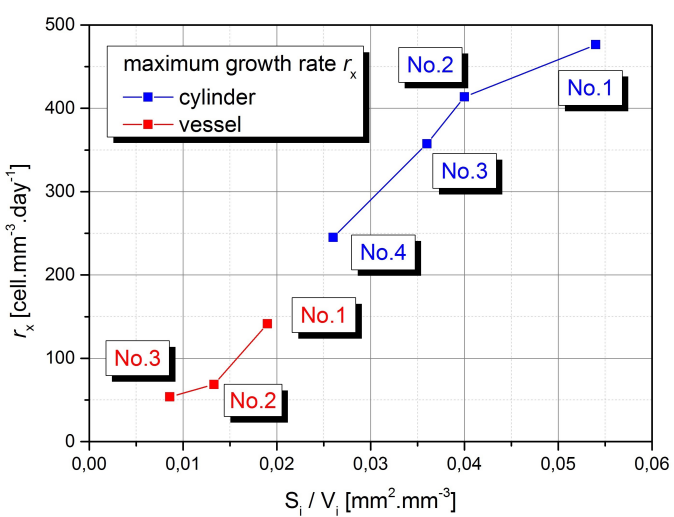

d)

FiguRE 8. Kinetics of biomass growth: a) in cylinders, b) in vessels, c) the effect of the volume of the photobiorector on the maximum growth rate, d) the effect of the ratio of the directly illuminated area to the volume of the photoreactor on the maximum growth rate.

$S_{i}$, the volume of the vessel $V_{i}$ (Figure 2 and the maximum growth rate $r_{x, \max }$ (Figure $8 \mathrm{~d}$ ). The results clearly show the strong influence of the area $S_{i}$ on which the light falls in relation to the volume $V_{i}$. And this applies equally to the mixed vessels and the unmixed cylinders.

(2.) Total gross biomass yield of biomass (algae). The overall yield of biomass $Y_{x / V}$ in the previous experiments was another evaluation of the experiments. It is a parameter that shows how much biomass has been cultivated in a given volume under the given operating parameters of the photoreactor. Due to the different sizes of the photoreactors and the volumes of the culture medium $V_{i}$, it is advantageous to compare the yield of biomass per unit volume:

$$
Y_{x / V}=\frac{\Delta x}{V_{i}} .
$$

By comparing the results in Figure 8 and Figure 9 it can be seen that by increasing the volume of the $V_{i}$ photoreactor vessel, the maximum growth rate of the biomass $r_{x, \max }$ decreases, but ultimately, the resulting $Y_{x / V}$ biomass yield increases. This is due to the fact that in the initial state at the beginning of the cultivation, when the biomass con- centration $x_{0}$ is the same in all the vessels, there is a larger number of individual microorganisms in the whole volume of the culture medium $V_{i}$ for large vessels than in the small ones. This means that the process in larger vessels runs at a lower growth rate $r_{x, \max }$ but a larger number of organisms $x_{i}$ are divided, which ultimately results in a higher biomass production $Y_{x / V}$.

Comparing the yields $Y_{x / V}$ from the individual vessels, the following can be seen: The highest yield was obtained from the largest cylinder with the volume $V_{4}$, although the maximum growth rate of biomass $r_{x, \max }$ was the smallest of all four cylinders. In the stirred vessels, the 3 liter volume has the highest yield.

If the fundamental methodology of the scale-up is to be compared, it is necessary to simultaneously observe the maximum growth rate of the biomass $r_{x, \max }$ and its total yield $Y_{x / V}$. These parameters are not proportional but have an opposite character. In order to create the optimal conditions for the growth of biomass, the photoreactor must have a small cross section so that the intensity of the illumination is as high as possible throughout the cross-section. However, the volume photoreac- 


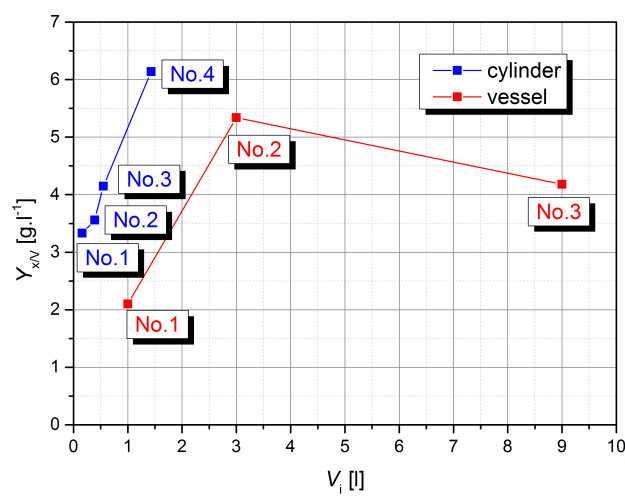

Figure 9. The resulting balance of wet centrifuged biomass after filtration relative to the unit volume of the culture medium.

tor per unit length is small and thus the resulting biomass yield also decreases.

Therefore, a compromise needs to be found. Figure 10 shows a compromise solution between the yield $Y_{x / V}$, maximum growth rate $r_{x, \max }$ and volume $V_{i}$.

For the cylinders (Figure 2a), point 1 is the compromise solution (Figure 10p. This gives a cylinder volume $V_{i}=0.84$ litres at which a growth rate $r_{x, \max }=321$ cell. $\mathrm{mm}^{-3}$.day ${ }^{-1}$ would be achieved. However, this is achieved when the cylinder geometry fulfils the condition $S_{i} / V_{i}=0.033 \mathrm{~mm}^{-1}$ (Figure 8d). At $L_{i} / d_{i}=4$ (Figure 2 the diameter of the cylinder should be $d_{i}=60.6 \mathrm{~mm}$ and the height of the level $L_{i}=291 \mathrm{~mm}$.

For the vessels (Figure $2 \mathrm{p}$ ), the compromise solution would be point 2 (Figure 10 ). The vessel volume is $V_{i}=1.44$ litres with the maximum growth rate of $r_{x, \max }=127$ cell. $\mathrm{mm}^{-3}$.day ${ }^{-1}$. The ratio between the illuminated area and the volume is then $S_{i} / V_{i}=0.017 \mathrm{~mm}^{-1}$ (Figure $8 \mathrm{~d}$ ). From these data, the diameter of the vessel is $d_{i}=117.6 \mathrm{~mm}$ and the height is $L_{i}=132.6 \mathrm{~mm}$. The Reynolds number $R e_{M}=27694$ must be used for mixing.

\section{Conclusion}

The experiments mentioned in this article are based on the process modelling theory. They were focused on examining the impact of geometrically similar photoreactors to examine the growth rate of biomass and the overall yield at the end of a particular growing period. The results show their opposite tendency. It is not possible to achieve a high growth rate of biomass as well as yield. Therefore, when designing photoreactors, it is necessary to optimize the relationship between these two parameters. This ensures the appropriate lighting conditions as well as an effective productivity per unit volume of the culture medium. In the case of tubular photobiorectors it, is necessary to ensure the flow of the medium in these tubes as

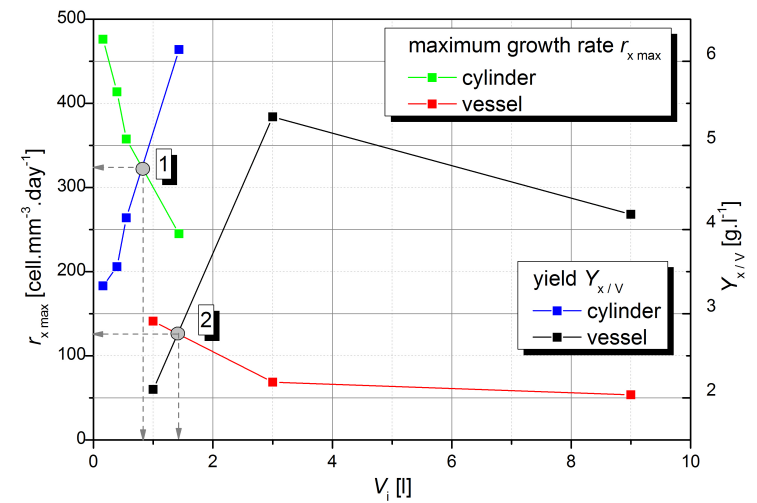

Figure 10. Optimizing the process parameters of a photoreactor.

well as stirring, in order to allow the light to be accessed uniformly to the individual algae. If the ratio between the biomass growth rate and the yield is not optimized, the energy supplied to the culture medium by the pump is not optimally utilized.

The experiments once again confirmed the wellknown fact that the rate of the growth of biomass significantly decreases with the increasing diameter of the photobiorector. Therefore, from the scale-up point of view, it is not advisable to increase the dimensions of a device by enlarging the diameter. It is advantageous to find an optimum tube diameter that ensures an optimal ratio between the illuminated surface and the volume of the device.

For the industrial applications, it is not necessary to immediately initiate a pilot plant photobioreactor. Economically and time wise, it is appropriate to examine the kinetics of the growth and yield of algae on a series of small models. Based on these results, a pilot plant will be built and then, an operation facility on an industrial scale as shown in Figure 1 can be designed. The procedure outlined in this paper demonstrates how the scale-up of photoreactors could be realized. The results show the non-linear behaviour of the system and that the modelling with the scaleup is problematic and needs to be given a sufficient attention.

\section{LIST OF SYMBOLS}

$d_{i} \quad$ Vessel diameter $[\mathrm{m}]$

$d_{f}$ Tube diameter of an industrial photobioreactor $[\mathrm{m}]$

$d_{m}$ Diameter of the agitator $[\mathrm{m}]$

$n$ Stirrer speed $\left[\mathrm{s}^{-1}\right]$

$r_{i} \quad$ Distance in polar coordinates $[\mathrm{m}]$

$r_{i}$ Growth rate $\left[\right.$ cell $\mathrm{mm}^{-3}$ day $^{-1}$ ]

$r_{x, \max }$ Maximum growth rate $\left[\right.$ cell $\mathrm{mm}^{-3}$ day $^{-1}$ ]

$t$ Time [s]

$x_{\mathrm{o}}$ Initial biomass concentration $\left[\right.$ cell $\left.\mathrm{l}^{-1}\right]$

$x_{i}$ Biomass concentration [cell $1^{-1}$ ]

$I$ Hourly incident photosynthetic radiation on a horizontal surface $\left[\mu \mathrm{E} \mathrm{m}^{-2} \mathrm{~s}^{-1}\right]$ 
$I_{\text {av }}$ Photosynthetically active hourly average irradiance inside culture $\left[\mathrm{HE} \mathrm{m}^{-2} \mathrm{~s}^{-1}\right]$

$I_{\mathrm{Bt}}\left(r_{i}, \varphi\right)$ Direct local hourly photosynthetically active irradiance inside vertical column $\left[\mathrm{HE} \mathrm{m}^{-2} \mathrm{~s}^{-1}\right]$

$I_{\mathrm{Dt}}\left(r_{i}, \varphi\right)$ Local hourly disperse photosynthetically active irradiance inside vertical column $\left[\mu \mathrm{E} \mathrm{m}^{-2} \mathrm{~s}^{-1}\right]$

$L_{i} \quad$ Height of the culture medium in the vessel $[\mathrm{m}]$

$P \quad$ Biomass productivity $\left[\mathrm{kg} \mathrm{m}^{-3} \mathrm{~s}^{-1}\right]$

$R$ Radius or hydraulic radius $[\mathrm{m}]$

$R e_{M}$ Reynolds number modified for mixing [1]

$S_{i} \quad$ The area affected by the sun's rays $\left[\mathrm{m}^{2}\right]$

$V_{i} \quad$ Volume of the culture medium $\left[\mathrm{m}^{3}\right]$

$Y_{x / V}$ Yield of biomass per unit volume $\left[\mathrm{kg} \mathrm{l}^{-1}\right]$

$\alpha$ Parameter [1]

$\varepsilon$ General angle [rad]

$\eta \quad$ Dynamic viscosity $[\mathrm{Pas}]$

$\varphi$ Angular position in polar coordinates [rad]

$\mu \quad$ Specific growth rate $\left[\mathrm{s}^{-1}\right]$

$\mu_{\max }$ Maximum specific growth rate $\left[\mathrm{s}^{-1}\right]$

$\rho$ Density $\left[\mathrm{kg} \mathrm{m}^{-3}\right]$

\section{ACKNOWLEDGEMENTS}

This work was supported by the Ministry of Education, Youth and Sports of the Czech Republic under OP RDE grant number CZ.02.1.01/0.0/0.0/16-019/0000753 "Research centre for low-carbon energy technologies".

This article was created with the support of the Ministry of Education, Science, Research and Sport of the Slovak Republic within the Research and Development Operational Programme for the project "University Science Park of STU Bratislava", ITMS 26240220084, co-funded by the European Regional Development Fund.

The authors wish to acknowledge the Scientific Grant Agency of the Ministry of Education, Science, Research and Sport of the Slovak Republic and the Slovak Academy of Sciences for the financial support of this research by grant VEGA 1/0276/17.

\section{REFERENCES}

[1] M. H. Wilson, J. Groppo, A. Placido, et al. Co2 recycling using microalgae for the production of fuels. Applied Petrochemical Research 4(1):41-53, 2014. DOI:10.1007/s13203-014-0052-3

[2] M. A. Borowitzka. High-value products from microalgae - their development and commercialisation. Journal of Applied Phycology 25(3):743-756, 2013. DOI:10.1007/s10811-013-9983-9.

[3] N. R. Moheimani, M. P. McHenry, K. de Boer, K. Bahri. Biomass and Biofuels from Microalgae. Springer International Publishing Switzerland, 2015. DOI:10.1007/978-3-319-16640-7.

[4] A. Melis, T. Happe. Trails of green alga hydrogen research - from hans gaffron to new frontiers. Photosynthesis Research 80:401-409, 2004. DOI:10.1023/B:PRES.0000030421.31730.cb

[5] S. Iersel, A. Flammini. Algae-based biofuels: applications and co-products. FAO Environmental and Natural Resources Service Series 44, 2010. ISBN 978-92-5-106623-2.
[6] P. Spolaore, C. Joannis-Cassan, E. Duran, A. Isambert. Commercial applications of microalgae. Journal Of Bioscience And Bioengineering 101(2):87-96, 2006. DOI:10.1263/jbb.101.87

[7] A. Richmond. Microalgae in human and animal nutrition. Handbook of microalgal culture. WileyBlackwell, Oxford, 2004. ISBN: 978-0-632-05953-9.

[8] O. Pulz, W. Gross. Valuable products from biotechnology of microalgae. Applied Microbiology and Biotechnology 65(6):635-648, 2004. DOI:10.1007/s00253-004-1647-x

[9] L. Brennan, P. Owende. Biofuels from microalgae - a review of technologies for production, processing, and extractions of biofuels and co-products. Renewable and Sustainable Energy Reviews 14:557-577, 2010. DOI:10.1016/j.rser.2009.10.009.

[10] C. Posten. Design principles of photo-bioreactors for cultivation of microalgae. Engineering in Life Science 9(3):165-177, 2009. DOI:10.1002/elsc.200900003

[11] E. M. Grima, F. G. A. Fernández, F. G. Camacho, Y. Chisti. Photobioreactors: light regime, mass transfer, and scaleup. Journal of Biotechnology 70:231-247, 1999 DOI:10.1016/S0168-1656(99)00078-4

[12] K. Kumar, C. N. Dasgupta, B. Nayak, et al. Development of suitable photobioreactors for co2 sequestration addressing global warming using green algae and cyanobacteria. Bioresource Technology 102:4945-4953, 2011. DOI:10.1016/j.biortech.2011.01.054

[13] http://www.fao.org/docrep/field/003/AC183E/ AC183E04.htm Accessed: 2017-08-15.

[14] http://www.engineeringtoolbox.com/ gases-solubility-water-d_1148.html. Accessed: 2017-08-15.

[15] http:

//www.k-state.edu/olsonlab/chlamy_media.html. Accessed: 2017-08-15.

[16] M. A. Scranton, J. T. Ostrand, F. J. Fields, S. P. Mayfield. Chlamydomonas as a model for biofuels and bio-products production. The Plant Journal 82:523-531, 2015. DOI:10.1111/tpj.12780

[17] E. Harris. The Chlamydomonas Sourcebook. 2008. ISBN 978-0-12-370873-1.

[18] L. Chriaštel, J. Soltes. Bioreaktor. Vydavatelstvo STU v Bratislave, 2003. ISBN 80-227-1843-2, In Slovak.

[19] E. Günerken, E. D' Hondt, M. H. M. Eppink, et al. Cell disruption for microalgae biorefineries. Biotechnology Advancesl 33:243-260, 2015. DOI:10.1016/j.biotechadv.2015.01.008

[20] V. Montalescot, T. Rinaldi, R. Touchard, et al. Optimization of bead milling parameters for the cell disruption of microalgae: Process modeling and application to porphyridium cruentum and nannochloropsis oculata. Bioresource Technology 196:339-346, 2015. DOI:10.1016/j.biortech.2015.07.075.

[21] T. Žáková. Modelovanie procesov v bioreaktoroch. 2017. Diploma thesis. STU in Bratislava, Faculty of Mechanical Engineering, Institute of Process Engineering. STU in Bratislava, Bratislava. In Slovak. 